\title{
The Contribution of Mechanical Spectroscopy to Understanding Grain Boundary Sliding
}

\author{
Daniele Mari ${ }^{a}$ *밍 \\ ${ }^{a}$ Faculté des sciences de base, École polytechnique fédérale de Lausanne, Lausanne - EPFL, \\ Switzerland
}

Received: May 25, 2018; Accepted: August 29, 2018

\begin{abstract}
This review paper shows that grain boundary sliding (GBS) is a general phenomenon occurring in all classes of inorganic materials: ceramics, metals and composite materials. The occurrence of relaxations attributed to GBS is also quite general and therefore the mechanical spectroscopy constitutes a sensitive and universal technique to study such phenomenon. GBS is widely observed in ceramics. It can be due to the presence of an amorphous layer between the grains as in zirconia or to dislocations, as in alumina. In each case, a high temperature GBS peak has been identified. In metals, GBS is observed in some deformed materials but the correlation of such phenomenon with internal friction peaks has been controversial. In 1941, C. Zener describes a geometrical model of GBS that could give rise to a relaxation mechanism. In 1947, Kê observed a large relaxation peak in polycrystalline aluminum. This peak being absent in single crystals, the relaxation was attributed to GBS. Today, the Zener model can still be used in most cases for relaxations occurring in the grain boundaries. Instead, according to the grain boundary type, the material or the temperature, either dislocations or the gliding of a disordered layer produce the grain boundary relaxations.
\end{abstract}

Keywords: Internal friction, ceramics, alloys, grain boundary migration.

\section{Introduction}

Grain boundary sliding (GBS) is a well-known mechanism leading to plasticity of materials such as ceramics, metals and composites. In fine grain ceramics, GBS constitutes the principal mechanism of deformation at high temperature ${ }^{1,2}$. This phenomenon has been attributed to the presence of a glassy layer between the grains ${ }^{3}$ both in oxides and nitrides. However, in ceramics that do not contain such layer, GBS has been attributed to dislocations emitted from the boundary ${ }^{4}$. In all cases, GBS must be associated with bulk deformation in order to keep the coherency of the material ${ }^{5}$. The presence of a phase different from that of the matrix in the grain boundary is also determinant for the deformation of composite materials such as cermets ${ }^{6}$. In particular, a change of the thermodynamical equilibrium under stress allows the infiltration of cobalt of WC boundaries in WC-Co based cemented carbides ${ }^{7}$. In metals, deformation by GBS is more rarely observed directly, even if it has been considered as a creep mechanism. Direct evidence of GBS has been reported mostly in $\mathrm{Zn}$ alloys ${ }^{8,9}$. However, many simulation studies have been published concerning nanocrystalline materials ${ }^{10}$. The mobility of grain boundaries, such as that of any other defect, can be evidenced by mechanical spectroscopy. This technique consists in measuring the phase lag $\tan (\phi)$ between a periodic stress $\sigma=\sigma_{0} \mathrm{e}^{i \omega t}$ with angular frequency $\omega$ and strain $\varepsilon=\varepsilon_{0} \mathrm{e}^{i(\omega t-\phi)}$.

Such a phase lag depends on the energy $W_{\text {diss }}$ dissipated in the material by defects that are moving under the action of the periodic stress:

"e-mail: daniele.mari@epfl.ch.

$$
\tan (\phi)=\frac{1}{2 \pi} \frac{W_{\text {diss }}}{W_{\text {el.max. }}}=\Delta \frac{\omega \tau}{1+(\omega \tau)^{2}}
$$

Where $W_{\text {el. } \max .}$ is the maximum elastic energy stored during a cycle. It can be shown ${ }^{11}$ that if $\tau$ is the characteristic relaxation time (no distribution), $\tan (\phi)$ displays a Debye peak as a function of angular frequency $\omega$ (equation 1 ). The amplitude of the relaxation is the factor $\Delta$ and the maximum of the peak attains a value of $\Delta / 2$. The main proof of the existence of grain boundary relaxations is generally the comparison of the spectrum of a single crystal with that of a polycrystal. This paper reviews the evidences of GBS at the atomic scale that have been observed by mechanical spectroscopy.

\section{Ceramics}

The first peak attributed to GBS in ceramics was reported by Lakki in polycrystalline zirconia $^{12}$ who elaborated a model based on GBS with a restoring force at triple points. However, the first comparison between a polycrystal and a single crystal was actually displayed by Donzel et al. ${ }^{13}$ as shown in Figure 1. By observing the figure 1, one could argue that a peak is present in the single crystal and therefore it could not be attributed to GBS. In reality, the peak of the single crystal is a point defect Zener peak that was attributed to $\mathrm{Ca}$ dopant with an activation energy of $422 \mathrm{~kJ} / \mathrm{mol}$ et an attempt frequency $f_{0}=10^{-14}$. The temperature and the activation energy distinguish the GB peak from the Zener peak. It is interesting that Donzel evidenced two GBS peaks 


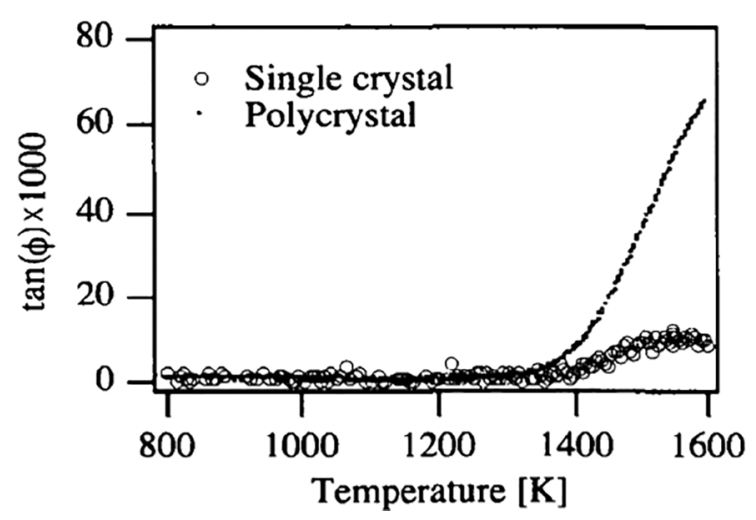

Figure 1. Comparison of temperature spectra between a $3 \mathrm{YT} \mathrm{ZrO} 2$ polycrystal and a $\mathrm{ZrO} 2-16 \mathrm{~mol} . \% \mathrm{CaO}$ single crystal

with more or less the same activation energy $(560 \mathrm{~kJ} / \mathrm{mole})$ : a first one at lower temperature that should be attributed to a glass transition in amorphous pockets at triple points and the second one, which is the true grain boundary peak. In high purity samples, only the true GBS peak is present. Since these peaks are located at very high temperature, isothermal frequency scans are not adapted to measure the activation energy since the calculation should be based on a few spectra. The method of the Master Curve was then used to measure activation energies. It consists in shifting the frequency spectra obtained in isothermal conditions in order to obtain a unique matching curve. The plot of $\delta(\log (f))$ vs. $\delta(1 / T)$ produces an Arrhenius plot from which the activation energy can be found. However, the fit is not straightforward. Mazaheri ${ }^{14}$ realized that after high temperature isothermal annealing, the level of internal friction in isothermal spectra was decreasing. He supposed that the strain depends on grain size according to a Dorn creep law ${ }^{15}$. Therefore, he was able to introduce in the master curve procedure a fit based on the grain size dependence, in addition to the temperature dependence. So, the isothermal spectra are not only shifted in frequency but also (vertically) according to $\log (\tan (\phi))$ :

$$
\delta(\log (\tan (\phi)))=-p \delta(\log (d))
$$

The grain size exponent $p$ is that found in the Dorn creep law. Then, adopting an Ostwald ripening power law for growth kinetics ${ }^{16}$ :

$$
d^{m}-d_{0}^{m} \approx d^{m}=k \cdot t
$$

The exponent $m=3$ is often taken for isotropic growth. Combining equations 2 and 3 , one can then expect an internal friction varying as :

$$
\delta(\log (\tan (\phi)))=-\frac{p}{m} \delta(\log (k \cdot t))
$$

Effectively a slope $\frac{p}{m}=0.3$ was found corresponding to an interface reaction growth ${ }^{17}$ with $p=1$. Such a slope becomes instead $\frac{p}{m}=0.53$ in alumina. This is close to $\mathrm{p}=2$ and $\mathrm{m}=3$, which is expected for dislocation climb. Effectively, in alumina grain boundaries are crystalline and no amorphous zone is found. The mechanism modeled by Lakki was indeed based on dislocation climb ${ }^{18}$.

\section{Cermets}

Cermets constituted by WC-Co or TiCN-Co are important materials for the manufacturing of cutting tools. Since the tool working conditions involve high temperatures, the mechanisms leading to plastic deformation have been investigated extensively ${ }^{6}$. GBS has been considered as the main mechanism of high temperature creep. In fact, GBS of WC grains was observed by markers in $\mathrm{WC}^{-} \mathrm{Co}^{19}$. But, in parallel with GBS, clear signs of cobalt infiltration of the grain boundaries are observed both in WC-Co and TiCN-Co ${ }^{7,20}$. At the temperature where those phenomena are observed, a high mechanical loss peak is shown by mechanical spectroscopy ${ }^{21}$ (Figure 2). The peak is very broad, which can be justified by the variety of grain boundary orientations. The peak was tentatively analyzed as the superimposition of 4 peaks ${ }^{22}$. In this case, the activation energies of such peaks are not anymore apparent. The amplitude of the 4 peaks varies as a function of the temperature, which can be correlated with the degree of infiltration (or segregation) of the cobalt into the WC interfaces.

\section{Metals}

In 1941, C. Zener ${ }^{23}$ predicted a grain boundary relaxation and made the first model of GBS in the anelastic limit, i.e. considering the grain boundaries pinned at triple points and sliding between. Such a model predicted a relaxation strength essentially depending on the Poisson ratio $v$. By taking $\nu=0.3$, the amplitude of the peak should be $\Delta=0.64$. In 1947 , T.S. Kê that was Research Associate in Zener's Institute of

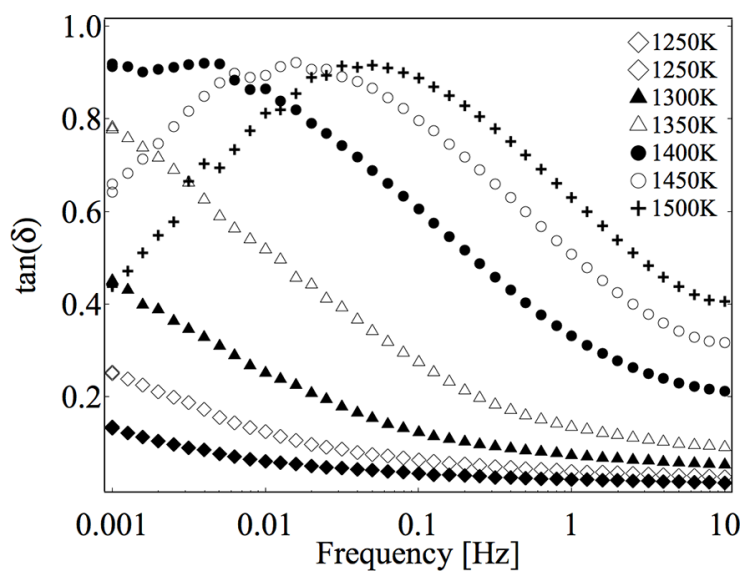

Figure 2. Isothermal frequency scans measured upon heating and colling in a WC-10vol\%Co with some Ta addition 
Metals in Chicago discovers a large relaxation with $\Delta=0.4$ at about $200{ }^{\circ} \mathrm{C}$ in aluminum ${ }^{24}$. The relaxation is absent in a single crystal and Kê deduces that grain boundaries must be responsible the relaxation (Figure 3). Moreover, by developing Zener's theory, he comes to the conclusion that the GBS peak amplitude should not depend on grain size while the relaxation time should increase linearly with grain size. Such trend corresponded to observations in the aluminum samples ${ }^{25}$. For almost 30 years, grain boundary relaxations were studied in Hefei where T.S. Kê had become Professor until some doubts were expressed in two groups in France, in Poitier by Woirgard et al. ${ }^{26,27}$ and in Lyon ${ }^{28,29}$. These authors show that by making accurate experiments on a single crystal, a peak can be found at the same temperature as the peak attributed to GBS. The peak found in the single crystal is always much smaller than that of polycrystalline samples. Those findings support the idea that dislocations are responsible for the GBS peak discovered by Kê. Another argument supporting the dislocation theory is the strong sensitivity of the peak to microstructure. It can be noticed that cold work modifies the peak both restoring $\mathrm{it}^{28}$ or decreasing $\mathrm{it}^{30}$. On the other hand, the presence of impurities clearly enhances the peak amplitude while shifting it at higher temperature ${ }^{31,32}$. This result is very important. In fact, it is regrettable that so many efforts have been devoted to studying very pure materials and in particular aluminum. It is very likely that in the case of aluminum, the peak is due to dislocations. But in very pure aluminum, the microstructure is very complex and only long and meticulous studies relating the microstructure and the internal friction spectrum subcomponents may be convincing. Instead, the use of alloys, even complex, often produces very stable and reproducible peaks.

An example was obtained with Ni20at. $\% \mathrm{Cr}^{33}$. The internal friction and elastic shear modulus temperature spectrum of polycrystalline Ni-20 at.\% Cr, obtained both

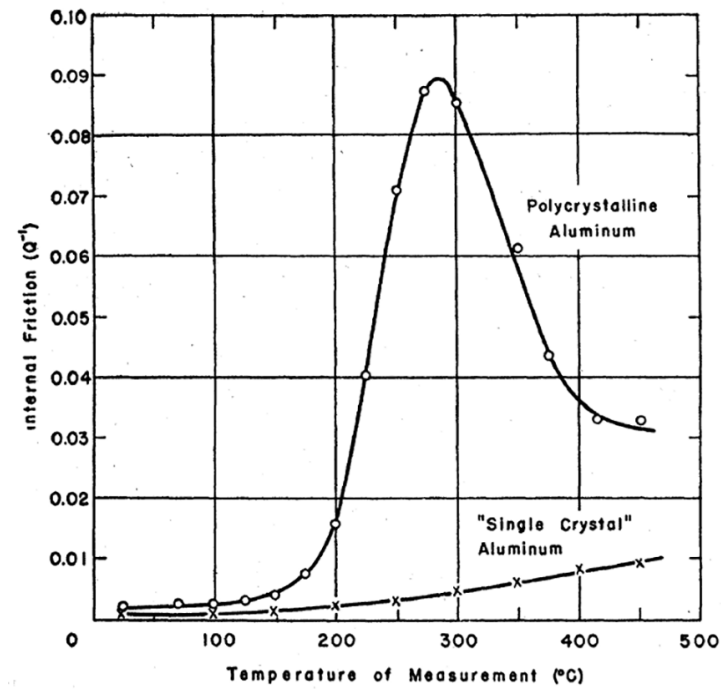

Figure 3. Grain boundary peak in aluminum as reported by $\mathrm{Ke}^{24}$ during heating and cooling, is presented in Figure 4. The spectrum mainly shows two internal friction peaks, marked $\mathrm{P} 1$ and $\mathrm{P} 2$, respectively, as well as a large thermal hysteresis between the curves obtained during heating and cooling. The same thermal cycle was applied to a single-crystal specimen (Figure 5). In this case, only the P1 peak is observed, whereas the peak P2 and the large thermal hysteresis are totally absent. This result eliminates the possibility for the peak P2 to be due to any dislocation mechanism inside the grains. Another experiment, (Figure 5) was performed on a $12 \%$ cold-rolled Ni-20at.\%Cr single crystal. Heating above the peak temperature $(1200 \mathrm{~K})$ leads to recrystallization. After two cycles, the internal friction spectrum was identical to that of the polycrystalline specimen shown in Figure 4. The hysteresis in the Ni-Cr peak is related to solutionprecipitation of chromium carbides that can be observed at grain boundaries. In order to confirm that the precipitation hinders the GBS, it was possible to produce a sample where no carbon was present and therefore no carbides. In such case, a stable GBS peak can be observed on heating and cooling. These experiments also show that probably a collective GBS phenomenon is involved.

More recently, a detailed study was developed on a gold alloy widely used in jewellery: Au10at $\% \mathrm{Cu} 30 \mathrm{at} \% \mathrm{Ag}$.

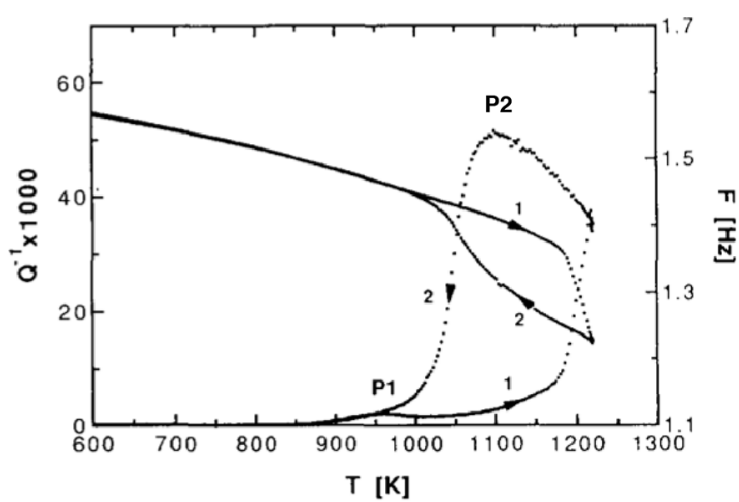

Figure 4. Hysteretic behaviour in Ni-20at.\%Cr. The peak P2 only appears if sample is heated above $1200 \mathrm{~K}$

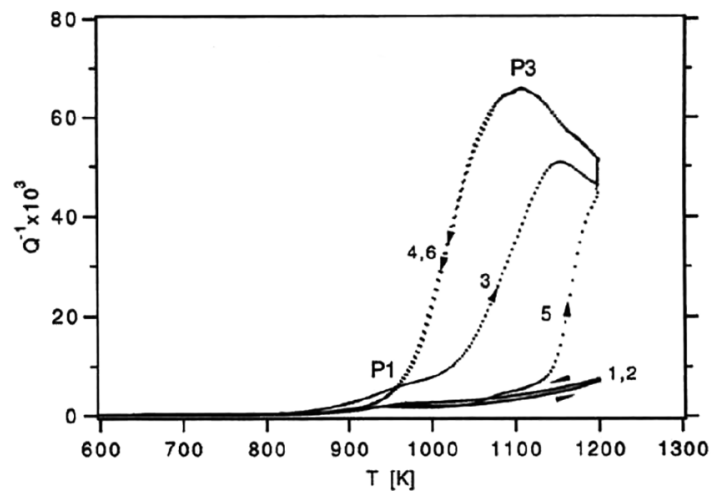

Figure 5. Spectra obtained in $\mathrm{Ni}-20 \mathrm{at} . \% \mathrm{Cr}$ starting from single crystal $(1,2)$, after $12 \%$ cold work at RT (3) and upon successive cooling after $6 \mathrm{~h}$ at $1200 \mathrm{~K} 4$. Curves 5 and 6 represent the following heating and cooling cycle 


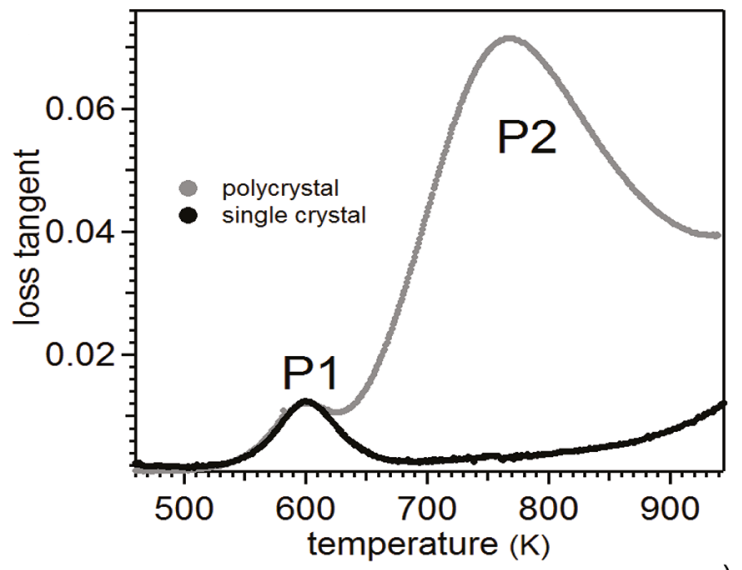

a)

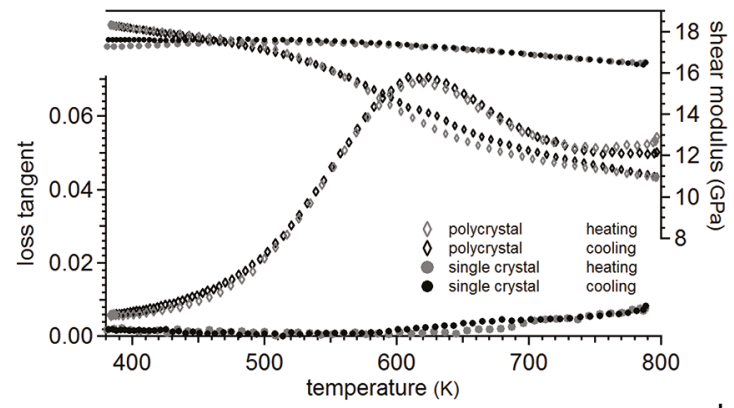

b)

Figure 6. a) Spectrum obtained in Au10at $\%$ Cu30at $\%$ Ag alloy at $0.5 \mathrm{~Hz}$. The peak P2 that only appears in the polycrystal is attributed to grain boundaries. b) In pure gold the peak appears at lower temperature, at about $600 \mathrm{~K}$

A broad peak P2 is found at about $750 \mathrm{~K}$ at $1 \mathrm{~Hz}$, but such peak is completely absent in single crystals ${ }^{34}$. The spectrum (Figure 6a) looks very similar to that of Ni-Cr with a Zener peak P1 that precedes on heating the GBS peak. However, the peak P2 is stable and no hysteresis has been found. Very accurate measurements of the activation energy give $2.08 \mathrm{eV}$ with an attempt frequency of $110^{14}$ $\mathrm{Hz}^{35}$. A similar peak is found in pure (4N) gold at $600 \mathrm{~K}$ in the polycrystalline state while the single crystal does not show any evidence of a peak in the same temperature region (Figure 6b). Since in this alloy the GBS peak is very stable, the authors investigated in depth the effect of grain size. Replicating the measurements by Cao described above, a monocrystalline 18-carat sample was cold-worked stepwise from $2 \%$ to $12 \%$ at room temperature ${ }^{36}$. The mechanical loss spectrum was then analyzed at each step. At low deformations, the exponential high temperature background increases. At a critical deformation of $10 \%$, a peak very close to that of the polycrystal appeared. Moreover, samples deformed between $2 \%$ and $8 \%$ evidence the existence of an intermediate stage. The high temperature peak, which can be evidenced only in frequency spectra, characterizes the mobility of low angle grain boundaries as proven by the comparison with an intentionally synthesized one. It is likely that the mobility of low angle grain boundaries is controlled by the movement of dislocations.

In $\mathrm{Au}-\mathrm{Cu}$ alloys, it is relatively easy to control the grain growth, which occurs at $950 \mathrm{~K}$ well above the peak temperature. In a study by Maier et al. ${ }^{34}$, a sample was cycled in temperature several times and annealed at 923 $\mathrm{K}$ or even $1000 \mathrm{~K}$ in order to obtain grain growth. At each cycle, a metallographic observation allows the grain size measurement. The variation of the peak relaxation time depended exactly as the inverse of the grain size as predicted by Zener. Instead the peak amplitude was independent of the grain size. These measurements prove unequivocally that the topological model proposed by Zener applies very well to polycrystalline homogeneous materials. At this point, one should recall that the behavior of a polycrystal with a bamboo structure, i.e. grain boundaries completely crossing the sample and parallel to the shear direction, is completely different: in this case the amplitude of the GBS peak should depend on the grain size density i.e. $A / V$ where $\mathrm{A}$ is the total surface of the grains and $\mathrm{V}$ is the sample volume. Kê had made the first experiments proving such a relation ${ }^{37}$. More recently two groups have worked on bi-crystals either made of aluminum ${ }^{38}$ or Au-Cu-Al ${ }^{39}$. By reducing the size of the sample it is very easy to control and to assess the grain boundary density. Clearly, in the case where grain boundaries are crossing the internal friction specimen to each end, the dependence on grain boundary density was fully proven.

All these data provide convincing phenomenological evidences that a grain boundary relaxation exists in metals. However, the microscopic mechanism leading to such relaxations are still controversial. Molecular dynamics simulation may lead to a deeper insight about those mechanisms. For example, simulation in Au polycrystals of a $\sum 5 \mathrm{~GB}$, undergoing shear deformation ${ }^{40}$ show that grain boundary migration coupled to sliding is found below 700K. Above $1000 \mathrm{~K}$, only GBS is observed. Two models, one based on migration and the other based on sliding can be developed. They provide expressions for the relaxation strength $\mathrm{D}$ and the relaxation time $t$. They are then compared to experimental measurements performed on polycrystal ${ }^{34}$. The observed grain size dependence of $\mathrm{D}$ and $\mathrm{t}$, i.e. $\mathrm{D}$ independent of grain size and $t$ linearly dependent on the inverse of grain size, support the sliding model against the migration one.

\section{Conclusions}

Exploring a wide range of materials from ceramics to metals and composites, grain boundary sliding is a mechanism that strongly affects plastic deformation. It is also clear that such mechanism of macroscopic deformation can be related to microscopic relaxations evidenced by Mechanical Spectroscopy. 
In ceramics, grain boundary sliding can be due either to the presence of an amorphous layer such as in $\mathrm{ZrO}_{2}$ or to dislocations such as in $\mathrm{Al}_{2} \mathrm{O}_{3}$.

The presence of the second phase in grain boundaries is critical for GBS in WC-Co composite materials. The change of the thermodynamical equilibrium at high temperature causes the cobalt infiltration of tungsten carbide grain boundaries. Grain boundary relaxation peaks are associated to this effect.

In metals, GBS is more rarely supported by macroscopic evidences. However, relaxations related to the presence of grain boundaries are widespread. The original geometrical mechanism of sliding imagined by Zener seems applicable in most cases. However, there is still a debate about the microscopic mechanisms. In fact, in very pure metals such as aluminum or copper, complex dislocation movements involving climb and glide, are likely to cause the GBS.

In alloys, instead a phase transition involving the formation of an amorphous phase could favor the glide of the boundary and once again justify the Zener model.

\section{References}

1. Nieh TG, Wadsworth J. Superplastic ceramics. Annual Review of Materials Science. 1990;20:117-140.

2. Wakai F. A review of superplasticity in $\mathrm{ZrO} 2$-toughened ceramics. British Ceramic Transactions. 1989;88(6):205208 .

3. Pharr GM, Ashby MF. On creep enhanced by a liquid phase. Acta Metallurgica. 1983;31(1):129-138.

4. Lartigue Korinek S, Dupau F. Grain boundary behavior in superplastic Mg-doped alumina with yttria codoping. Acta Metallurgica et Materialia. 1994;42(1):293-302.

5. Ashby MF, Verrall RA. Diffusion-accommodated flow and superplasticity. Acta Metallurgica. 1973;21(2):149-163.

6. Mari D. Mechanical Behavior of Hardmetals at High Temperature. In: Sarin VK, ed. Comprehensive Hard Materials. Oxford: Elsevier; 2014. p. 405-421.

7. Östberg G, Andrén HO. Microstructural changes during wear by plastic deformation of cemented carbide and cermet cutting inserts. Metallurgical and Materials Transactions A. 2006;37(5):1495-1506.

8. Kaibyshev OA. Fundamental aspects of superplastic deformation. Materials Science and Engineering: A. 2002;324(1-2):96102.

9. Sheikh-Ali AD. Coupling of grain boundary sliding and migration within the range of boundary specialness. Acta Materialia. 2010;58(19):6249-6255.

10. Van Swygenhoven H, Derlet PM. Grain-boundary sliding in nanocrystalline fcc metals. Physical Review B. 2001;64(22):224105.

11. Nowick AS, Berry BS, eds. Anelastic Relaxation in Crystalline Solids. New York: Academic Press; 1972. 677 p.
12. Lakki A, Schaller R, Nauer M, Carry C. High temperature superplastic creep and internal friction of yttria doped zirconia polycrystals. Acta Metallurgica et Materialia. 1993;41(10):28452853.

13. Donzel L. Intra and Intergranular high temperature mechanical loss in Zirconia and Silicon Nitride. [Thesis]. Lausanne: Ecole Polytechnique Fédérale de Lausanne; 1998.

14. Mazaheri M, Mari D, Schaller R. High temperature mechanical spectroscopy of yttria stabilized zirconia reinforced with carbon nanotubes. Physica Status Solid (a). 2010;207(11):2456-2460.

15. Mukherjee AK, Bird JE, Dorn JE. Experimental Correlations for High-Temperature Creep. Transactions of American Society for Metals. 1969;62:155-179.

16. Wagner $\mathrm{C}$. Theorie der Alterung von Niederschlägen durch Umlösen (Ostwald-Reifung). Zeitschrift für Elektrochemie, Berichte der Bunsengesellschaft für physikalische Chemie. 1961;65(7-8):561591.

17. Raj R, Chyung CK. Solution-precipitation creep in glass ceramics. Acta Metallurgica. 1981;29(1):159-166.

18. Lakki A, Schaller R, Carry C, Benoit W. High temperature anelastic and viscoplastic deformation of fine-grained $\mathrm{MgO}$-doped $\mathrm{Al}_{2} \mathrm{O}_{3}$. Acta Materialia. 1998;46(2):689-700.

19. Mari D, Marti U, Silva PC. A new photolithographic technique to detect the local deformation of materials: application to WC-Co composites. Materials Science and Engineering: A. 1992;158(2):203-206

20. Bolognini S, Feusier G, Mari D, Viatte T, Benoit W. TiMoCN based cermets: high temperature deformation. International Journal of Refractory Metals and Hard Materials. 2003;21(1-2):19-29.

21. Mari D. Understanding the mechanical properties of hardmetals through mechanical spectroscopy. Materials Science and Engineering: A. 2009;521-522:322-328.

22. Mari D, Buss K, Benoit W. Phase transitions at grain boundaries in WC-Co and internal friction peak analysis. Materials Science and Engineering: A. 2006;442(1-2):179-183.

23. Zener C. Theory of Elasticity of Polycrystals with Viscous Grain Boundaries. Physical Review. 1941;60(12):906-908.

24. Kê TS. Experimental Evidence of the Viscous Behavior of Grain Boundaries in Metals. Physical Review. 1947;71(8):533-546.

25. Kê TS. Stress Relaxation across Grain Boundaries in Metals. Physical Review. 1947;72(1):41-6.

26. Woirgard J, Amirault JP, de Fouquet J, eds. A Dislocation Model for Grain Boundary Peaks in Pure Face Cnetered Cubic Metals. In: Proceedings of 5 th Internernational Conference on Internal Friction and Ultrasonic Attenuation in Crystalline Solids; 1973 Aug 27-30; Aachen, Germany. Springer-Verlag; 1975.

27. Rivière A, Woirgard J. Etude du frottement interieur a haute temperature de polycristaux et de monocristaux de nickel de haute purete. Scripta Metallurgica. 1983;17(3):269-273.

28. Nó ML, Esnouf C, San Juan J, Fantozzi G. Internal friction at medium temperature in high purity aluminium and its relation with the microstructure-I. Acta Metallurgica. 1988;36(4):827836. 
29. Nó ML, EsnoufC, Juan JS, Fantozzi G. Internal friction at medium temperature in high purity aluminium and its relation with the microstructure-II. Acta Metallurgica. 1988;36(4):837-845.

30. Shi Y, Cai B, Kong QP, Cui P, Gottstein G. The effect of Ga on internal friction of pure $\mathrm{Al}$ before and after deformation. Journal of Materials Science. 2003;38(9):1895-1899.

31. San Juan ML. Etude par frottement intérieur de la mobilité des dislocations aux températures supérieures a 0,3 Tf dans l'aluminium haute pureté. [Thesis]. Lyon: Institut National des Sciences Appliquées de Lyon; 1985.

32. Kê TS, Cui P. Effect of solute atoms and precipitated particles on the optimum temperature of the grain boundary internal friction peak in aluminium. Scripta Metallurgica et Materialia. 1992;26(9):1487-1492.

33. Cao B, Schaller R, Benoit W, Cosandey F. Internal friction associated with grain boundaries in Ni-Cr alloys. Journal of Alloys and Compounds. 1994;211-212:118-123.

34. Maier AK, Tkalcec I, Mari D, Schaller R. Grain boundary relaxation and grain growth in 18-carat yellow gold alloy. Scripta Materialia. 2012;66(6):374-377.
35. Audren AK. Grain Boundary Relaxation in 18-carat Gold Alloys. [Thesis]. Lausanne: Ecole Polytechnique Fédérale de Lausanne; 2014.

36. Maier AK, Tkalcec I, Mari D, Schaller R. Grain boundary formation stages in a deformed yellow gold single crystal studied by mechanical spectroscopy. Materials Science and Engineering: A. 2013;560:466-472.

37. Kê TS, Zhang BS. Contribution of Bamboo Boundaries to the Internal Friction Peak in Macrocrystalline High-Purity Aluminium. Physica Status Solid (a). 1986;96(2):515-525.

38. Jiang WB, Kong QP, Cui P. Further evidence of grain boundary internal friction in bicrystals. Materials Science and Engineering: A. 2010;527(21-22):6028-6032.

39. Maier AK, Mari D, Tkalcec I, Schaller R. Grain boundary relaxation in yellow gold bi-crystals. Materials Science and Engineering: A. 2015;632:43-49.

40. Maier AK, Mari D, Tkalcec I, Schaller R. Theoretical modelling of grain boundary anelastic relaxations. Acta Materialia. 2014;74:132-140. 